Artículo

\title{
Evaluación de un programa experiencial de educación parental: la perspectiva de los educadores como agentes de cambio
}

\author{
Susana Torío López*, Carmen M. Fernández García y María de las Mercedes Inda Caro \\ Departamento de Ciencias de la Educación, Universidad de Oviedo, Oviedo, Asturias, España
}

\section{INFORMACIÓN DEL ARTÍCULO}

\section{Historia del artículo:}

Recibido el 6 de febrero de 2014

Aceptado el 18 de mayo de 2015

On-line el 7 de agosto de 2015

\section{Palabras clave:}

Evaluación de formadores

Programas de educación parental

Formación de padres y madres

Corresponsabilidad familiar

Intervención familiar

\begin{abstract}
R E S U M E N
En la formación de padres y madres en programas de tipo experiencial, modelo al que se adscribe el programa de educación parental que se presenta, "Construir lo cotidiano", juega un importante papel la persona responsable del grupo, el formador/educador que implementa el mismo, entendiendo a este como un agente de cambio. En estas páginas queremos resaltar la importancia de dicho agente de intervención e ilustrar la reflexión con los resultados obtenidos en la aplicación del programa puesto en marcha durante el curso académico 2010/2011 en 4 centros educativos en los niveles de Educación Infantil y Primaria del Principado de Asturias. En el estudio participan 34 personas (16 parejas de familias nucleares y 2 familias monoparentales) así como 8 formadores ( 2 personas en cada centro) encargados de su desarrollo. Los resultados cuantitativos y cualitativos de la evaluación, desde la perspectiva de los educadores, ponen de manifiesto que el programa ha resultado efectivo para sus participantes y se han percibido cambios en las familias. Las valoraciones de los formadores son fundamentales para conocer mejor el desarrollo del programa y constituyen una herramienta básica para regular el clima emocional y los aspectos motivacionales del grupo.
\end{abstract}

(C) 2015 Instituto de Ciencias de la Educación de la Universidad de Oviedo. Publicado por Elsevier España, S.L.U. Este es un artículo Open Access bajo la licencia CC BY-NC-ND (http://creativecommons.org/licenses/by-nc-nd/4.0/).

\section{Evaluation of an experiential program for parents: Educators as agents of change}

\section{A B S T R A C T}

In experiential parents training programs, such as "Building the everyday», the role of the person who is responsible for the group is crucial. Furthermore, the trainer/educator who implements it, is seen as an agent of change. In this article, emphasis is placed on the importance of the trainer, and illustrates this idea with the results obtained in the implementation of the training program. This took place in the academic year 2010/2011 in four schools of Pre-school and Primary Education in the Principality of Asturias. Thirty four people participated in the study (16 pairs of nuclear families and two single parent families), as well as 8 trainers ( 2 people in each school). Quantitative and qualitative evaluation results obtained from the educators show that the program has been effective for its participants, and that changes have been noticed in all the families. These trainers' perceptions are essential to understand the development of the program, and they are also a basic tool to regulate the emotional climate and the motivational aspects of the group.

(C) 2015 Instituto de Ciencias de la Educación de la Universidad de Oviedo. Published by Elsevier España, S.L.U. This is an open access article under the CC BY-NC-ND license (http://creativecommons.org/licenses/by-nc-nd/4.0/).

\section{Introducción}

Los programas educativos grupales para fomentar competen-

* Autor para correspondencia: Universidad de Oviedo, C/ Aniceto Sela s/n, 03300 Oviedo.

Correo electrónico: storio@uniovi.es (S. Torío López). cias parentales en materia de corresponsabilidad aluden al hecho de que las actividades domésticas y de cuidado de las personas con quienes se convive sean compartidas por personas de ambos 
sexos. Por tanto, responden a un reparto equitativo de las responsabilidades sociales, familiares y profesionales, de tal forma que el sustento económico y las tareas domésticas sean asumidas por todas las personas que componen la unidad familiar.

El programa de educación parental que lleva por título «Construir lo cotidano» (Torío, Peña, Rodríguez, Fernández, Molina, Hernández e Inda, 2013) se centra en abordar la corresponsabilidad familiar con el objetivo de proporcionar la oportunidad de unas relaciones más igualitarias y nuevos modelos de socialización en la crianza y educación de los hijos e hijas. Se trata, por tanto, de hacer visible la necesidad de un reparto equilibrado de las tareas domésticas más tradicionales pero también de otras vinculadas a la planificación y dirección doméstica, al cuidado de los menores y al trabajo emocional (puede verse una síntesis del programa y estructura en Torío, Peña, Rodríguez, Fernández y Molina, 2010). Siguiendo a Vosler (1996), ayudar a las familias a identificar y organizar sus recursos disponibles puede ser una buena manera de sobrellevar la sobrecarga de roles. Así, cuando las familias poseen los recursos suficientes pero estos no son utilizados, puede ser útil trabajar las creencias y preferencias con respecto al trabajo familiar y los roles de género, así como dotarlas de nuevos patrones de comportamiento y organización.

Se trata de un programa de formación de tipo "experiencial» que concibe el contexto de intervención como un escenario sociocultural de construcción compartida del conocimiento cotidiano (Máiquez, Rodrigo, Capote y Vermaes, 2000 Martín-Quintana et al., 2009; Rodrigo, Correa, Máiquez, Martín y Rodríguez, 2006; Torío, Peña y Hernández, 2012). Los programas que parten de este modelo: «... tienen en cuenta las creencias o las ideas previas de los padres y las madres, ya que a partir de estas y de las situaciones cotidianas, y mediante un proceso inductivo de construcción del conocimiento cotidiano-experiencial, van a ir construyendo su conocimiento» (Martín-Quintana et al., 2009, p. 127). Esto supone que la tarea principal de los padres y las madres es identificar, reflexionar y analizar las propias ideas, creencias, sentimientos y acciones en las diferentes situaciones cotidianas. En efecto, partir de lo que hacen diariamente, de su modelo educativo, y no de lo que debería ser.

Este objetivo se consigue a través del intercambio con otras familias embarcadas en la misma tarea, ya que entre todos cuentan con un potencial de recursos y destrezas de gran utilidad (Máiquez et al., 2000). En este modelo de formación, por contraposición a un modelo académico (adquisición de conceptos sobre el desarrollo y la educación en un escenario de aprendizaje formal) o a un modelo técnico (adquisición de técnicas y procedimientos basados en la modificación de conducta, en el aprendizaje social en un escenario de aprendizaje experto), los padres y las madres se sienten activos y protagonistas, lo que implicaría que la intervención debe enfocarse hacia el desarrollo de sentimientos de confianza en su capacidad para educar a sus hijos e hijas.

A diferencia de otros programas, el programa «Construir lo cotidiano" trata de abordar la formación parental en un período relativamente más tranquilo que el de la adolescencia, trabajando con familias con hijos e hijas de edades comprendidas entre los $0 \mathrm{y}$ 12 años, en una etapa en la que las prácticas educativas y las rutinas están menos asentadas que unos años más tarde. No pretende ser una recopilación de recetas sino de ideas y, cada familia, con su experiencia, las concretará en cada momento con las respuestas adecuadas.

En una formación experiencial, el educador que desarrolla el programa debe adoptar un modelo de usuario o de personas legas entrenadas para guiar la dinámica, otorgando el máximo respeto hacia las iniciativas personales de las familias, sin cuestionarlas, e incrementando el grado de libertad en la toma de decisiones: "los padres no son ayudantes (...) el profesional se encarga de ofrecer a los padres una gama de opciones y la información necesaria para que autónomamente puedan realizar una selección. Esta forma de actuar lleva implícito el reconocimiento de los padres como personas que conocen su propia situación, tienen experiencia y son competentes» (Máiquez et al., 2000, pp. 47-50).

Por otro lado, en este modelo, la actuación del profesional se fundamenta en la negociación entendida como un proceso mediante el cual se llega a acuerdos aceptables, dentro de una relación de respeto mutuo. Su intervención quedaría definida por las siguientes tareas (Máiquez et al., 2000, pp. 52-53): a) como facilitador en las formas de verbalización que emplean los padres y las madres para referirse a situaciones educativas, a sus concepciones y a los comportamientos; b) como fuente de información al proporcionar modelos alternativos que no hayan salido espontáneamente en el discurso explícito de los padres y las madres; c) como apoyo y ayuda en el proceso de intercambio y negociación de los puntos de vista ante las distintas situaciones educativas y d) como regulador del clima emocional del grupo para que el autoconcepto parental, las relaciones interpersonales y los aspectos motivacionales sean los adecuados.

Por tanto, como parece desvelar lo expuesto, la actuación del educador que aplica el programa resulta fundamental. Como se ha dicho, frente a la idea de "técnico» o "experto», el formador pasará a construir el conocimiento como un miembro más, regulando las aportaciones de los padres y madres y centrándolas en la dinámica del grupo. Ello implica que, además de formarse en los temas básicos del programa, debe tener unas cualidades y aptitudes especiales para desempeñar este trabajo: debe estar preparado ante la aparición de mecanismos de defensa de los padres y madres, es necesario que sepa transitar de una fase a otra del programa, que garantice que se respeta el turno de palabra, debe saber escuchar a los que participan, debe mostrar capacidad para reconocer y tratar las emociones, saber evaluar las necesidades de las familias, etc.

De igual modo, los guías que implementen el programa deben estar atentos a los diferentes problemas que pueden minar su éxito. Por ejemplo, los conflictos que puedan surgir en cuanto a puntos de vista y valores procedentes de familias que son diferentes y cuyas normas y valores pueden ser contrarios; la pérdida de interés por parte de los asistentes con el tiempo (índice de desgaste); la preocupación por la intimidad; la monopolización de conversación por parte de determinadas parejas, entre otros.

Los objetivos principales de este trabajo son: conocer qué aspectos permiten un mejor seguimiento de un programa orientado a padres y madres (objetivo 1) y determinar qué procesos experienciales contribuyen a un óptimo desarrollo del programa y a la posterior transferencia a la realidad familiar (objetivo 2). Estos 2 objetivos serán analizados desde la perspectiva de los educadores y las educadoras del programa.

\section{Método}

\section{Participantes}

Las personas que han desarrollado el programa ( 6 mujeres y 2 varones), cuya media de edad es 46,5 años, tienen estudios universitarios superiores del ámbito de la educación con experiencia en la dinámica de grupos y en la intervención socioeducativa.

El programa de educación parental "Construir lo cotidiano» combina la información teórica en el ámbito de la educación familiar con el apoyo en la progresividad de la tarea educativa de las familias; es decir, aporta información sobre diferentes temáticas relevantes de la educación familiar, pero parte del análisis y comprensión de las propias problemáticas de los padres y madres para hacer aflorar las concepciones subyacentes sobre cómo educar para facilitar el cambio de actitudes y conductas. Para tal fin, en el perfil de los formadores se acentúa el entrenamiento en 
Tabla 1

Características de las familias evaluadas

\begin{tabular}{|c|c|c|c|c|c|c|}
\hline Colegios & $\begin{array}{l}\text { Edad media en el } \\
\text { nacimiento del } \\
\text { primer hijo/a }\end{array}$ & $\begin{array}{l}\text { Promedio del } \\
\text { número de } \\
\text { hijos/a }^{\text {b }}\end{array}$ & $\begin{array}{l}\text { Promedio de la } \\
\text { edad del primer } \\
\text { hijo/a en años }\end{array}$ & $\begin{array}{l}\text { Promedio de la } \\
\text { edad del segundo } \\
\text { hijo/a en años }\end{array}$ & 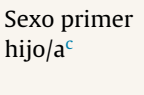 & $\begin{array}{l}\text { Sexo segundo } \\
\text { hijo/a }\end{array}$ \\
\hline Colegio 1 & 37,50 & 1 & 6 & - & Varón & - \\
\hline Colegio $2^{\mathrm{a}}$ & 31 & 1 & 6,4 & 6 & Varón & Varón \\
\hline Colegio 3 & 38 & 2 & 6,25 & 4 & Mujer & Varón \\
\hline Colegio 4 & 32,60 & 1 & 9,5 & 8 & Varón & Mujer \\
\hline
\end{tabular}

a En este centro había una familia con 3 hijos. Dos varones y una mujer. La niña pequeña de 4 años.

b Prueba de Kruskal-Wallis para establecer las diferencias de medias $\left(\chi^{2}=10,78 ; p=0,01\right)$.

c Se presenta la moda en esta variable.

habilidades comunicativas (capacidad de escucha y saber mostrar aceptación, ser facilitador de las formas de verbalización de los padres y madres para llegar a identificar, analizar, reflexionar sobre sus sentimientos, creencias, reflexiones sobre su modelo educativo), habilidades interpersonales y relacionales (persona abierta, flexible, empática), habilidades para el manejo de grupos así como la adherencia o fidelidad a los contenidos del programa y la compenetración con el compañero o compañera de sesión.

En la aplicación del programa en los 4 centros participantes se ha establecido un acompañamiento en pareja, es decir, acuden un educador responsable (encargado del programa en el centro) y otro educador acompañante (realiza tareas de observación, síntesis, reparto de materiales, control de tiempos, etc.).

El grupo de padres y madres con los que han trabajado los formadores ha estado constituido por 34 personas procedentes de 2 colegios públicos y 2 centros concertados de Oviedo y Gijón. Como puede apreciarse, debido a las características y enfoque del programa, es imprescindible que los grupos sean reducidos, con los que se trabaja desde una perspectiva que se aproxima más al estudio de casos que al manejo de grandes grupos, en los que el educador se centra en la transmisión unidireccional de información. Digamos pues, que el número de participantes se encuentra condicionado por las exigencias de nuestros objetivos y método de trabajo. La distribución por género ha sido de 16 varones $(47,1 \%)$ y 18 mujeres (52,9\%). La presencia de 2 mujeres más se explica debido a la participación de 2 familias monoparentales. La media de edad es de 42,88 en los varones y de 42,28 en las mujeres. La edad media en la que se han convertido en padres ha sido los 35 años. Parece interesante señalar que, por centros, se han encontrado diferencias estadísticamente significativas $\left(\chi^{2}=10,78 ; p=0,01\right)$, con una media de 31 años de edad, seguidos por 32,6; 35,17 y 37,50.

Como se puede observar en la tabla 1 la media de hijos o hijas en los 4 centros es de uno por familia, con la excepción en uno de los centros donde ha participado una familia con 3 descendientes. Respecto al sexo del primer descendiente, en 3 de los centros el valor más encontrado ha sido el de los varones, y en uno, las niñas. Esta tendencia se observa, también, respecto al segundo hijo. Respecto a la edad del primer hijo no se han encontrado diferencias estadísticamente significativas, circunstancia que sí se aprecia en la edad del segundo descendiente: prueba de Kruskal-Wallis $\left(\chi^{2}=7 ; p<0,05\right)$.

Para finalizar, en el nivel de estudios, se han encontrado diferencias estadísticamente significativas $\left(\chi^{2}=16,82 ; p<0,01\right)$ : el $35,3 \%$ de los participantes han alcanzado los estudios universitarios medios, $25,5 \%$ universitarios superiores; $20,6 \%$ formación profesional; $8,8 \%$ educación secundaria, $5,9 \%$ estudios primarios y un $2,9 \%$ han obtenido el nivel de doctor.

\section{Instrumentos de evaluación}

Para el desarrollo de estas páginas, se utilizarán los datos obtenidos de los 2 instrumentos de evaluación diseñados específicamente para el programa.
1. Cuestionario de fin de sesión (Torío, Peña, Fernández, Inda y Rodríguez, 2013b): se trata de un instrumento que cumplimenta el educador responsable, al final de cada sesión. Es un cuestionario formado por 16 ítems. Once de ellos ofrecían la opción de respuesta dicotómica, 1 (no), 2 (sí), y los 5 restantes presentaban 4 alternativas de respuestas desde 1 (nunca) hasta 4 (siempre). Para el análisis cuantitativo, estos 5 ítems se dicotomizaron en 1 y 2.

El contenido del cuestionario tiene por objetivo que el formador realice una autoevaluación de su trabajo durante la sesión. Se pregunta, en primer lugar, sobre aspectos organizativos $y$ metodológicos: la claridad en la presentación de los objetivos de la sesión; el grado de operativización en la presentación, mediante ejemplos; la explicación de las actividades que deben realizar durante la sesión así como en la tarea para casa; las dificultades observadas para seguir el manual del educador, etc. Asimismo, se pide que valore, además, el clima de trabajo que se ha mantenido durante la sesión y la comunicación socioafectiva con las familias: cómo se ha manejado la adherencia al programa por parte de las familias participantes, si ha sido capaz de hacerse con la confianza del grupo, de responder a las preguntas y comentarios planteados por el grupo, etc. Finalmente, respecto a los participantes, se reflexiona sobre la posibilidad de transferir los conocimientos a la vida diaria, el nivel de satisfacción y de utilidad percibidos por las familias, así como la posibilidad de constatar cambios y su prolongación en el tiempo.

2. "Guía de notas» (Torío et al., 2013b): se trata de un instrumento de carácter abierto que debe ser elaborado por el educador acompañante. Constituye un instrumento de evaluación cualitativa, semejante al de las notas de campo "condensadas" (Spradley, citado en Valles, 1997), en el que el educador realiza una descripción y observación sobre cómo ha tenido lugar el desarrollo de la sesión. Su carácter abierto permite que cada uno de los educadores pueda plasmar la diversidad de situaciones, imprevistos, percepciones del grupo, participación e intercambio de opiniones que tienen lugar en cada uno de los grupos de padres y madres recogiendo: «juicios, opiniones, sospechas, dudas, reflexiones que el investigador añade a la mera información descriptiva» (Rodríguez, Gil y García, 1996, p. 203). Por su carácter cualitativo y sus posibilidades para la recogida de todas las experiencias particulares que pueden darse, se trata de un material muy valioso en la evolución y seguimiento del proceso formativo de los participantes.

Este instrumento se concibe desde el concepto de una evaluación formativa, que posibilite realizar un seguimiento permanente del programa con el objetivo de lograr un mayor éxito. Ello exige prestar atención a los problemas que puedan ir surgiendo e ir introduciendo las sugerencias o modificaciones necesarias. 


\section{Procedimiento}

El enfoque del programa exige un trabajo del equipo estructurado y planificado. Cada una de las 9 sesiones que componen el programa (hay que incluir una décima de cierre, en la que se trabajan los aspectos que han quedado menos claros, que demandan mayor profundización o aquellos cuyos compromisos son más difíciles de cumplir) ha sido preparada por los miembros del equipo de investigación de manera individual y, con posterioridad, se pactaron reuniones semanales por parte de los 2 personas educadoras implicadas (responsable y acompañante). El objetivo de este encuentro, previo al desarrollo del programa, no es otro que revisar y concretar la actuación de cada uno de ellos en el trabajo con las familias. Por tanto, cada uno de los miembros del equipo ha tenido asignadas tareas específicas con el objetivo de realizar un buen ajuste con el marco teórico y pedagógico del programa.

Al finalizar cada una de las sesiones el educador responsable cumplimenta el cuestionario de fin de sesión y el educador acompañante recoge en la hoja de «notas» aspectos significativos, impresiones, que hayan ocurrido en el desarrollo de la sesión, las percepciones del grupo, el grado de participación, etc.

\section{Análisis de datos}

Para analizar cuáles son los aspectos que más contribuyen a un seguimiento óptimo del programa, desde la perspectiva de los educadores (objetivo 1 ) se consideró necesario realizar análisis cuantitativos. El análisis cuantitativo empleó la prueba no paramétrica de Kendall para muestras relacionadas. La elección de esta técnica estadística permite analizar si existen diferencias dentro de los niveles de la variable "cuestionario de fin de sesión». Se selecciona esta prueba no paramétrica por tratarse principalmente de una muestra reducida, menor de 30 personas, y no garantizarse el cumplimiento de los criterios paramétricos para poder realizar una ANOVA de medidas repetidas. Por otro lado, este test estadístico proporciona, también, el coeficiente de Kendall, valor W. Dicho valor señala la proporción de las diferencias encontradas en cada uno de los niveles de la variable evaluada. Al tratarse de un estadístico cuyos valores oscilan entre 0 y 1 , cuanto más elevado es su valor mayores diferencias se encuentran entre los niveles de la variable. Si los estadísticos de esta prueba resultan significativos, se procederá a realizar el test no paramétrico de Wilcoxon para 2 muestras relacionadas con la consiguiente corrección de Bonferroni. Además se calculará el tamaño de los efectos siguiendo las indicaciones de Grisson y Kim (2012, pp. 172-173), donde $\mathrm{PS}_{\text {dep }}$ se define como la probabilidad de superioridad, permitiendo calcular el tamaño del efecto en los diseños de medidas relacionadas y $\mathrm{PS}_{\mathrm{dep}}=$ número de comparaciones positivas obtenidas en la prueba de Wilcoxon/número total de comparaciones. Como indica la American Psychological Association es recomendable calcular el tamaño de los efectos que ejerce una intervención educativa sobre los participantes (Wilkinson, 1999).

Para el segundo objetivo, el análisis de los procesos experienciales que mejor contribuyen al desarrollo del programa y a su transferencia a la realidad familiar, se consideró emplear una metodología cualitativa. La fuente principal de información ha sido el instrumento "guías de notas». Una vez finalizada cada una de las sesiones y en un plazo no superior a $24 \mathrm{~h}$ el educador acompañante debía redactar por escrito a partir de sus notas "condensadas» sus notas «expandidas» o informe de la sesión, en las que debe detenerse y ampliar todos los detalles (Spradley, citado en Valles, 1997). En dichas transcripciones debía ser recogida toda la información relevante no solo desde un punto de vista verbal sino también no verbal, de modo que posibilite completar una imagen lo más fidedigna posible del trascurso de la sesión. Dicha transcripción era posteriormente puesta en común con el educador principal y en las reuniones de preparación de las siguientes sesiones. Para el análisis de esta información cualitativa ha sido necesario revisar las transcripciones elaboradas por cada uno de los educadores en 3 niveles diferenciados: a) clarificación temática de las ideas verbalizadas (tomando como referencia las categorías obtenidas en el marco teórico del estudio y prestando especial atención a lo referido a la transferencia a la realidad); b) descripción del contenido de las transcripciones (de manera particular, otorgando protagonismo a las semejanzas y diferencias obtenidas entre los distintos grupos de trabajo) y, finalmente, c) interpretación teórica del contenido descrito.

\section{Resultados}

Evaluación del desarrollo del programa por parte de los formadores

El test de Kendall muestra que existen diferencias estadísticamente significativas entre los 16 niveles del instrumento "cuestionario de fin de sesión", $\chi^{2}(15, n=19)=153,44, p<0,001$, siendo el coeficiente $\mathrm{W}$ de Kendall $=0,54$. Por ello, se ha procedido a analizar entre qué niveles del instrumento de evaluación del educador se concentran las diferencias. En la tabla 2 se pueden ver los porcentajes de respuesta en cada uno de los niveles. En 3 ítems las frecuencias de respuesta están por debajo de 30. Si se analizan estos ítems, se ve que la valoración de estos aspectos en las primeras sesiones del programa es difícil de hacer por parte de los formadores, ya que el programa todavía se encuentra en sus estadios iniciales. Los datos de la mediana muestran que las diferencias se centran en el ítem que evalúa «conseguir confianza del grupo», en el que la mediana es igual a 1. El test de Wilcoxon ha revelado diferencias estadísticamente significativas con el resto de las variables del instrumento del educador y un tamaño de los efectos entre 0,52 y 0,86. Esta diferencia es entendible ya que el ítem evalúa "ha sido difícil conseguir la confianza del grupo». A su vez, dicho ítem sirve como elemento de referencia para la validez del instrumento. En este sentido, hay que destacar un ítem, con el que no se encontraron diferencias estadísticamente significativas ("participantes consideran útiles para la vida familiar la actividades para casa»). La ausencia de diferencias y la disminución del valor del tamaño de los efectos están indicando la necesidad de revisar esta cuestión. Este resultado cuantitativo se completa más adelante con los análisis cualitativos.

Análisis de los procesos experienciales que están determinando el seguimiento del programa desde la percepción de los formadores

Realizando un análisis más detallado de los aspectos organizativos y metodológicos del programa, se puede destacar que es a partir de la quinta sesión cuando los educadores valoran que su explicación ha sido siempre clara y concisa. En las 4 primeras sesiones las valoraciones se han repartido, al 50\%, entre "casi siempre» $\mathrm{y}$ «siempre».

Los formadores han indicado que en la mayoría de las sesiones han aportado ejemplos de las ideas principales. Veamos un ejemplo en las «notas» del educador acompañante (EA):

EA 3: "...valoran la posibilidad de hablar en la dinámica, "las ideas de todos puestas en común” -comentan los padres-, etc. ha sido una sesión en la que han participado mucho» (3. a sesión).

En muy pocas ocasiones se valora negativamente el establecimiento de ejemplos de las ideas principales. Solo en las primeras sesiones («Modos de educar en familia» $\mathrm{y}$ "Las normas son importantes para la vida familiar») y, en la séptima ("Cuidar la 
Tabla 2

Análisis de las respuestas del instrumento del educador al finalizar cada sesión

\begin{tabular}{|c|c|c|c|c|c|}
\hline & No $\%(n)$ & Sí \% (n) & Md & $\mathrm{W}^{\mathrm{a}}$ & ps \\
\hline Claridad de los objetivos & 0 & $100(36)$ & 2 & $-5,3^{*}$ & 0,86 \\
\hline Explicación clara & $2,8(1)$ & $97,2(35)$ & 2 & $-5,5^{*}$ & 0,86 \\
\hline Definición cuidadosa & $2,8(1)$ & $97,2(35)$ & 2 & $-4,8^{*}$ & 0,80 \\
\hline Ejemplos para ideas principales & $5,6(2)$ & $94,4(34)$ & 2 & $-4,9^{*}$ & 0,83 \\
\hline Actividades de la sesión explicadas claramente & 0 & $100(36)$ & 2 & $-5,3^{*}$ & 0,86 \\
\hline Actividades para casa de la sesión explicadas correctamente & $19,4(7)$ & $80,6(29)$ & 2 & $-5^{*}$ & 0,69 \\
\hline Seguir instrucciones del manual & $11,1(4)$ & $88,9(32)$ & 2 & $-4,5^{*}$ & 0,80 \\
\hline Conseguir clima de trabajo & $5,6(2)$ & $94,4(34)$ & 2 & $-4,6^{*}$ & 0,86 \\
\hline Aumento del interés en el programa & $2,9(1)$ & $97,1(34)$ & 2 & $-4,4^{*}$ & 0,83 \\
\hline Conseguir confianza del grupo & $88,9(32)$ & $11,1(4)$ & 1 & & \\
\hline Responder adecuadamente dudas del grupo & $2,8(1)$ & $97,2(35)$ & 2 & $-4,9^{*}$ & 0,86 \\
\hline Participantes consideran útiles los contenidos del programa & $8,8(3)$ & $91,2(31)$ & 2 & $-5,1^{*}$ & 0,88 \\
\hline Participantes consideran útiles las actividades & $8,8(3)$ & $91,2(31)$ & 2 & $-4,7^{*}$ & 0,82 \\
\hline Participantes consideran útiles para la vida familiar las actividades para casa & $44,8(13)$ & $55,2(16)$ & 2 & $-2,4$ & 0,52 \\
\hline Cambios en los participantes & $12(3)$ & $88(22)$ & 2 & $-4,02^{*}$ & 0,76 \\
\hline Cambios en el tiempo & $9,1(2)$ & $90,9(20)$ & 2 & $-3,6^{*}$ & 0,82 \\
\hline
\end{tabular}

ps = tamaño de los efectos.

a Test de Wilcoxon, comparación con «conseguir confianza del grupo». Se aplicó la corrección de Bonferroni $=$ alpha $\times$ número de comparaciones. $\mathrm{p}<0,001$

comunicación: base de una buena relación familiar») se encuentran respuestas que indican que «a veces» les ha costado poner ejemplos.

Respecto a las instrucciones del manual del educador, entre uno y 2 monitores han indicado que "a veces» han tenido dificultades en la segunda sesión (las normas), en la séptima y octava (la comunicación). Así es constatado por una educadora:

EA 4: "Intentamos seguir el guion de la actividad o, al menos dar respuesta a las preguntas que se proponen, pero resulta complejo, puesto que las familias desean saber cómo poner las normas y límites y comienzan a dar ejemplos de sus casos particulares... Es complicado reconducirlas..." (4. ${ }^{\text {a }}$ sesión).

Respecto a la utilidad de los contenidos, la percepción del educador ha sido positiva en todas las sesiones:

EA 3: «... se observa cómo en cada sesión ha ido aumentando la confianza, espontaneidad y la implicación. Ello quizá podría deberse también a la valoración positiva que están haciendo del programa» (sesión $6 \cdot^{\mathrm{a}}$ ).

Referente a las actividades del programa y, a pesar de haber sido los valores más pequeños en el tamaño de los efectos (tabla 2), cabe precisar que en las sesiones segunda (las normas), sexta (desencuentros y el reparto en la familia) y séptima (la comunicación), los educadores han percibido que los participantes no vislumbraban la utilidad de dichas tareas para su vida familiar. En la pregunta donde se valora si la explicación de las actividades propuestas para casa se ha realizado de manera clara, se encuentran respuestas negativas únicamente en las 5 primeras sesiones.

Los formadores han conseguido un buen clima de trabajo especialmente a partir de la tercera sesión. La capacidad de los monitores para responder a las dudas planteadas por el grupo ha tenido una respuesta entre "casi siempre» $\mathrm{y}$ "siempre».

EA 1: «Todos los participantes han participado sin dificultad, han preguntado muchas dudas y el ambiente ha sido muy bueno» (sesión 7. ${ }^{\mathrm{a}}$ ).

EA 3: «Las evaluaciones de fin de sesión reflejan el agrado de la dinámica y clima de trabajo» (sesión 2. ${ }^{\mathrm{a}}$ ).

La capacidad del monitor por lograr la confianza del grupo se consiguió de manera general relativamente pronto, si bien en las 2 primeras sesiones algunos grupos fueron más reticentes. En 3 de los 4 grupos participantes no ha sido difícil hacerse con la confianza del grupo (88,9\%: no y 11,1\%: sí). Así es manifestado en las notas de fin de la primera sesión por varios guías acompañantes:
EA 3: "Todos sin excepción han mostrado un alto grado de sinceridad y compromiso. Las intervenciones han sido distendidas y fluidas, hasta el punto de que la dinámica de grupos ha pasado muy rápido» (sesión 1 . $^{\mathrm{a}}$ ).

EA 1: «Ha sido positivo el tiempo que se ha dado a las parejas para que se conozcan y se sientan cómodas comunicándose con los demás miembros del grupo. Han sido capaces de transmitir sus aciertos, las dificultades que se encuentran en su vida familiar diaria, además de cómo se sienten» (sesión $1 .^{\mathrm{a}}$ ).

Por el contrario, en uno de los grupos, se percibe un sentimiento diferente y así es relatado por la educadora acompañante:

EA 4 "participación escasa, no acaban de «lanzarse» a hablar. Pienso que tienen miedo a la réplica, de ser cuestionados» (1. a sesión).

Con posterioridad, en la sesión $3 .^{a}$, la educadora escribe en sus notas:

EA 4: «Entran en el aula con otro semblante, más abiertos y se puede observar un ambiente mucho más cálido del vivido en la primera sesión. Me llama la atención sobre todo la actitud de A., pues entra en el aula contento, riendo y dispuesto a aportar y participar en las actividades...» (sesión 3. ${ }^{\mathrm{a}}$ ).

Conseguir progresivamente que vaya aumentando el interés de los participantes por el programa $(97,1 \%$, sí) ha sido señalado por ambos formadores en cada grupo de trabajo.

EA 3: "...cómo cada día se aprecia en mayor medida la implicación directa de los padres por 2 razones: la espontaneidad de sus comentarios y observaciones, y las cada vez más abundantes referencias inmediatas y directas a sus situaciones personales y familiares reales (...), han aflorado cuestiones de la dinámica familiar concretas, cómo nos organizamos, qué echamos en falta, hemos analizado nuestro comportamiento, se han transmitido sentimientos, etc.» (sesión 4. ${ }^{\mathrm{a}}$ ).

Las familias muestran especial interés en la realización por escrito de la tarea para casa. Muchas de ellas suponen el establecimiento de un compromiso para la semana o la puesta en práctica de los aprendizajes de la sesión.

EA 2: «Comenzamos la dinámica, con la revisión de la tarea que tenían para casa, preguntando a cada uno de los asistentes al respecto y. .. cabe decir que todos los miembros de cada una de las familias traían la tarea por escrito» (sesión 2. ${ }^{\mathrm{a}}$ ). 
La observación de cambios en los participantes ha sido indicada por los formadores a partir de la cuarta sesión. Se encuentran respuestas negativas, en esta pregunta, en las 3 primeras sesiones (esto explica la disminución en el tamaño de los efectos, tabla 2, en esta variable). En su mayor parte, los educadores ven avances y compromisos a lo largo de las sesiones. En la última sesión, uno de los guías acompañantes señala aspectos como los siguientes:

EA 2: «Todos realizan una valoración positiva y cómo han notado cambios en sus relaciones. I. habla de los cambios visibles en J. C. reflexiona cómo en el día a día se acuerda e intenta poner en práctica lo que han ido viendo en el programa» (sesión 10. ${ }^{\mathrm{a}}$ ).

Pero no estamos exentos de dificultades. Veamos, algunas reflexiones:

EA 4: «...tengo la sensación de que algunos participantes van recogiendo ideas, pero sin unir unas con otras (...). Pienso también que es ahora cuando el grupo empieza a "despegar", con su diversidad, pero apoyándose unos en otros y viendo el interés que tiene para su vida personal lo que en el programa se les plantea. La parte negativa de esto es que estamos en la séptima sesión y tan solo quedan 2 más con contenidos a explicar» (sesión 7. ${ }^{\mathrm{a}}$ ).

En todo caso, en la última sesión, la educadora acompañante concluye:

EA 4: "La satisfacción percibida es alta y el aprovechamiento medio-alto. A pesar de que tal vez ha resultado difícil que integren y extrapolen los contenidos abordados, al menos sí han reflexionado sobre la importancia de implicar a todos los miembros del hogar en las tareas domésticas, del diálogo, de la negociación y de los acuerdos. Cada participante ha pensado y proyectado en su vida aquello que sesión tras sesión íbamos trabajando y desmenuzando» (sesión 10. ${ }^{\mathrm{a}}$ ).

En la última pregunta del cuestionario, sobre si los cambios se prolongarán en el tiempo, a partir de la sexta sesión hay una unanimidad en los educadores en la respuesta afirmativa. En la primera sesión, los educadores no realizaron una valoración en este aspecto, en la segunda y tercera sesión, las respuestas se reparten entre el sí el no, al 75 y $25 \%$, respectivamente. En la cuarta sesión, el porcentaje de respuesta se divide al 50\%. En su mayor parte, los formadores acompañantes consideran que los compromisos acordados en el programa pueden prolongarse en el tiempo dentro de la dinámica familiar.

EA 1: «Esperamos que, en algunos casos, se prolonguen en el tiempo los cambios que ya han empezado a incorporar. En el caso de una de las parejas tenemos más dudas de si el programa habrá conseguido modificar alguna de las pautas familiares (se observa claramente cómo uno de los miembros de la pareja se encuentra poco dispuesto al cambio a pesar de que la otra parte lo reclama pública y permanentemente)» (sesión 10.a ).

\section{Discusión y conclusiones}

Ser padres y madres es una tarea evolutiva para la cual se requiere una amplia variedad de experiencias, que no se aprenden a resolver en entornos académicos ni de manera rápida. Además, no hay una única solución que se pueda aplicar a todos los casos ya que existen múltiples formas válidas de llevarlas a cabo en función de la multiplicidad de personas y situaciones. Como Triana y Rodrigo (2010) manifiestan: «se necesita la actuación específica de profesionales especializados que puedan orientar (...), a aquellas familias que estén encontrando dificultades en definir su proyecto educativo por diversas circunstancias...» (p. 121). Podemos decir, que la aplicación del programa ha tenido una buena acogida y aceptación por parte de las familias: les ha aportado aprendizajes útiles y ha requerido por parte de los educadores un alto grado de adaptación a las particularidades de cada grupo de padres, hecho constatado en las reuniones desarrolladas periódicamente por los educadores. En este sentido, el tamaño de los efectos está mostrando la efectividad del programa en sus destinatarios, a través de la percepción de los formadores. Bien es verdad que el cálculo del tamaño de los efectos se emplea para evaluar si un programa o intervención produce cambios en los destinatarios, y en este trabajo se están presentando las evaluaciones de los educadores, no obstante, desde la filosofía del programa, esta información también es indicativa de la efectividad del programa, ya que el rol del educador tiene un papel importante en el seguimiento y resultados del programa.

La evaluación de los cuestionarios de los educadores responsables así como las «notas» del formador acompañante han aportado información básica para conocer cómo se ha desarrollado la implementación del programa, como así ha sido constatado en otras investigaciones que abordan la evaluación de los programas de formación parental desde esta misma perspectiva (Amer y Vives, 2013; Klimes-Dougan et al., 2009; Orte, Ballester y March, 2013; Pascual, Amer y Fernández, 2012; Small, Cooney y OĆonnor, 2009). Esta información contribuye a la mejora de futuras implementaciones del programa y, de hecho, ya se han incorporado algunas modificaciones a partir de estos resultados.

Los formadores perciben que las familias participantes valoran tanto los aspectos formales del programa como los aspectos relacionales generados en las dinámicas. El hecho de la participación voluntaria, así como la atención recurrente y continua a las dudas y casos concretos planteados por las familias, ha contribuido de manera decisiva a esta valoración positiva. Todo ello ha ayudado a que las familias no perciban el proceso como «un» programa, sino como «su» programa.

Los formadores, además de ser fuente de información, ayuda y apoyo (Cataldo, 1991; Máiquez et al., 2000; Martínez González, 2009; Muñoz Silva, 2005; Orte et al., 2013), han sido los encargados de regular el clima emocional y participativo del grupo. Ha resultado fundamental establecer un clima de confianza mutua entre todos los participantes en el programa y posibilitar un ambiente que permita intervenir de forma abierta y sincera; es decir, alcanzar una reflexión cooperativa durante las sesiones. Ha constituido, por tanto, un apoyo y ayuda en el proceso de intercambio y negociación de los puntos de vista, más guiado al principio y mucho más fluido a medida que las sesiones han ido avanzando.

Un aspecto que se subraya en las distintas investigaciones son las habilidades requeridas a los formadores. Small et al. (2009) consideran claves las habilidades interpersonales y, en la misma línea, Klimes-Dougan et al. (2009) señalan que estas destrezas son más importantes incluso que la experiencia en la aplicación del programa. Igualmente, los formadores deben saber generar un clima de confianza agradable, deben ser capaces de mantener la atención y la cohesión grupal, deben manejar los grupos, deben saber trabajar en equipo, deben mostrar empatía e inteligencia emocional (Amer y Vives, 2013; Cataldo, 1991; Triana y Rodrigo, 2010). Dichos rasgos del facilitador se encuentran presentes en los resultados siguientes:

- Han sido una ayuda en la construcción de significados al regular y planificar las experiencias y comportamientos verbalizados.

- Han sido capaces de canalizar la atención hacia los aspectos relevantes y han sabido reorientar al grupo en caso de desvío.

- Han ofrecido un espacio de intercambio que ha facilitado la expresión de las preocupaciones y vivencias.

En suma, las contribuciones y valoraciones de los formadores responsables son fundamentales para conocer mejor el desarrollo del programa, aunque con esta información, únicamente, no se puede realizar una evaluación de objetivos con relación a si se han 
producido cambios positivos en las familias. Para tal fin, el programa cuenta con una evaluación previa (pretest) al comienzo y una evaluación final (postest) al finalizar el programa. Es importante formar a los educadores para mediar en los procesos de cambio, fomentar la colaboración y cohesión grupal. Así mismo, la combinación de análisis cuantitativos y cualitativos ha permito obtener resultados más completos. Sin embargo hay que señalar, como una de las limitaciones de este trabajo, el tamaño reducido de la muestra. Este es un hándicap condicionado por la necesidad de contar con la presencia de ambos miembros de la pareja para la participación en el programa, pero, no obstante, es también una de las novedades introducidas en el ámbito de los programas de educación parental.

\section{Conflicto de intereses}

Los autores declaran no tener ningún conflicto de intereses.

\section{Referencias bibliográficas}

Amer, J. y Vives, M. (2013). The importance of facilitators in family evidence-based prevention programs. In Paper presented at the Strengthening Family Strategy Online Conference. University of Balearic Islands (Spain), 7th-20th December, 2013.

Cataldo, C. Z. (1991). Aprendiendo a ser padres: conceptos y contenidos para el diseño de programas de formación de padres. Madrid: Visor.

Grisson, R. y Kim, J. (2012). Effect size measures that go beyond comparing two averages. In Effect size for research. Univariate and multivariate application. New York: Routledge.

Klimes-Dougan, B., August, G. J., Chih, Y., Lee, S., Realmuto, G., et al. (2009). Practitioner and site characteristics that relate to fidelity of implementation: The early risers prevention program in a going-to-scale intervention trial. Professional Psychology: Research and Practice, 40(5), 467-475.

Máiquez, M. L., Rodrigo, M. J., Capote, C. y Vermaes, I. (2000). Aprender en la vida cotidiana. Un programa experiencial para padres. Madrid: Visor.

Martín-Quintana, J. C., Byme, S., Máiquez, M. L., Rodríguez, B., Rodrigo, M. J. y Rodríguez, G. (2009). Programas de educación parental. Intervención psicosocial, 18(2), 121-133.

Martínez González, R. A. (2009). Programa-guía para el desarrollo de competencias emocionales, educativas y parentales. Madrid: Ministerio de Sanidad y Política Social.
Muñoz Silva, A. (2005). La familia como contexto de desarrollo infantil. Dimensiones de análisis relevantes para la intervención educativa y social. Portuaria, 5(2), 147-162.

Orte, C., Ballester, L. y March, M. X. (2013). El enfoque de la competencia familiar. Una experiencia de trabajo socioeducativo con familias. Pedagogía social. Revista Interuniversitaria, 21, 13-37.

Pascual, B., Amer, J. y Fernández, C. (2012). Evaluación del proceso de aplicación de un programa de competencia familiar en el ámbito de Servicios Sociales: la perspectiva de los educadores. In Universidad de Barcelona/SITE (Coord.), XII Congreso Internacional de Teoría de la Educación: Autonomía y responsabilidad. Contextos de aprendizaje y educación en el siglo XXI. Universidad de Barcelona.

Rodrigo, M. J., Correa, A. D., Maíquez, M. L., Martín, J. C. y Rodríguez, G. (2006). Family preservation services in Canary Island: Predictors of the efficacy of a parenting program for families at-risk of social exclusion. European Psychologist, 11(1), 57-70.

Rodríguez, G., Gil, J. y García, E. (1996). Aspectos básicos sobre el análisis de datos cualitativos. In Metodología de la investigación cualitativa. Málaga: Aljibe.

Small, S., Cooney, S. y O'Connor, C. (2009). Evidence-informed program improvement: Using principles of effectiveness to enhance the quality and impact of family-based prevention programs. Family Relations, 58, 1-13.

Torío, S., Peña, J. V., Rodríguez, M. C., Fernández, C. M. y Molina, S. (2010). Hacia la corresponsabilidad familiar: Construir lo cotidiano. Un programa de educación parental. Educatio Siglo XXI, 28(1), 85-108.

Torío, S., Peña, J. V. y Hernández, J. (2012). Primeros resultados de la aplicación y evaluación de un programa de educación parental: Construir lo cotidiano. Teoría de la Educación. Educación y Cultura en la Sociedad de la Información, 13(2), 343-368.

Torío, S., Peña, J. V., Rodríguez, C., Fernández, C. M., Molina, S., Hernández, J. y Inda M. M. (2013). Construir lo cotidiano: programa de educación parental. Barcelona: Octaedro.

Torío, S., Peña, J. V., Fernández, C., Inda, M. y Rodríguez, M. C. (2013). Pervivencias, fortalecimientos y cambios de las prácticas educativas familiares. In Paper presented at the Strengthening Family Strategy Online Conference. University of Balearic Islands (Spain), 7th-20th December, 2013.

Triana B. y Rodrigo M.J. (2010). Modelos y estrategias de intervención ante la diversidad familiar. En: E. Arranz Freijo y A. Oliva Delgado (Coord.), Desarrollo psicológico en las nuevas estructuras familiares (pp. 121-142). Madrid: Pirámide.

Valles, M. S. (1997). Técnicas de observación y participación: de la observación participante a la investigación-acción participativa. In Técnicas cualitativas de investigación social. Reflexión metodológica y práctica profesional. Madrid: Síntesis.

Vosler, N. R. (1996). New approaches to family practice: Confronting economic stress. London: Sage.

Wilkinson, L., \& The task force on statiscal inference APA Board of Scientific Affairs. (1999). Statiscal methods in psychology journals. Guidelines and explanations. American Psychologist, 54(8), 594-604. 